\title{
Microglial activation and recruitment, but not proliferation, suffice to mediate neurodegeneration
}

\author{
AD Rogove ${ }^{1,2,3}, \mathrm{~W} \mathrm{Lu}^{1,3}$, and SE Tsirka ${ }^{\star, 1}$ \\ 1 Department of Pharmacological Sciences, University Medical Center at Stony \\ Brook, Stony Brook, NY 11794-8651, USA \\ 2 MSTP Program, University Medical Center at Stony Brook, Stony Brook, NY \\ 11794-8651, USA \\ ${ }^{3}$ These authors contributed equally to the work \\ * Corresponding author: SE Tsirka, Dept. of Pharmacological Sciences, \\ University Medical Center at Stony Brook, Stony Brook, NY 11794-8651, USA. \\ Tel: (631) 444-3859; Fax: (631) 444-3218; E-mail: stella@pharm.sunysb.edu
}

Received 12.10.01; revised 13.12.01; accepted 31.1.02

Edited by CJ Thiele

\begin{abstract}
Microglial activation occurs during excitotoxin-induced neurodegeneration. We have reported that microglia can exhibit neurotoxic behaviors after injection of excitotoxins into the hippocampus. It is not known, however, whether microglial proliferation, which is part of the activation response, is required for neurodegeneration to be observed, or whether activation of the pre-existing resident microglia suffices. Using osteopetrotic (op/op) mice, in which injuryinduced microglial proliferation does not take place, we demonstrate that only the microglia initially residing in the CNS are adequate to promote neurodegeneration. Our data suggest that there is a threshold at which a maximal microglial contribution to neurotoxicity is observed. This threshold appears to be sufficiently low, such that activation of just $40 \%$ of the microglia present in wild-type mice serves to trigger neurodegeneration. Furthermore, since the decrease in microglial numbers coincides with a decrease in tissue plasminogen activator's activity, we suggest that tissue plasminogen activator can be used as a marker for microglial proliferation.

Cell Death and Differentiation (2002) 9, 801-806. doi:10.1038/ sj.cdd. 4401041
\end{abstract}

Keywords: microglia; neurotoxicity; osteopetrotic mouse; tissue plasminogen activator

Abbreviations: tPA, tissue plasminogen activator; op, osteopetrotic; CSF-1, macrophage colony stimulating factor

\section{Introduction}

When an excitotoxin is introduced into the mammalian hippocampus, neuronal death soon follows. This neuronal injury is accompanied by activation of the resident microglial cells. ${ }^{1}$ Onset of the microglial activation process is followed by their migration to the site of injury, local proliferation, changes in gene expression, presentation of class II major histocompatibility antigens, and phagocytosis. We and others have shown that microglia can exhibit neurotoxic properties when neuronal injuries are elicited by local injections of excitotoxins, ${ }^{1,2}$ autoimmune inflammation, ${ }^{3}$ or ischemia. ${ }^{4,5}$ Cytokines and neurotoxins secreted by microglia may help regulate the responses to injury in the central nervous system. ${ }^{6-8}$

In mice deficient for tissue plasminogen activator (tPA), a secreted serine protease normally present in the brain, hippocampal neurons are resistant to excitotoxic glutamate analogs and the microglia display attenuated activation. ${ }^{9}$ This attenuation is evident both as a decrease in the number of activated microglia present and as a decrease in the levels of expression of various microglial markers and cytokines. $^{10}$ Evidence in wild-type mice suggests that additional TPA is generated by microglia shortly after their activation and this tPA contributes to the ensuing neurotoxicity. ${ }^{2}$ Accordingly, strategies to decrease the amount of tPA released for the purpose of achieving neuroprotection could include blocking microglial activation, and specifically microglial recruitment or proliferation. In this study, we describe experiments designed to evaluate if a decrease in the number of activated microglia reduces excitotoxic neural injury using the mutant osteopetrotic (op/ op) mouse.

Op/op is a spontaneous mutation in C57BI6 mice that results in a deficiency of the macrophage colony stimulating factor (CSF-1) gene product. A single base pair insertion within this gene causes a frameshift mutation and creates a truncated protein that is non-functional. ${ }^{11}$ In cell culture, microglia isolated from op/op mice do not proliferate unless the medium is supplemented with CSF-1. ${ }^{12}$ Furthermore, microglial proliferation is dramatically reduced in the nucleus of the transected facial nerve after axotomy in op/op mice. ${ }^{13}$ However, the microglia that were present following axotomy in op/op mice displayed similar changes in morphology and gene expression as control op heterozygous $(o p /+)$ mice, indicating that they do respond to activation signals from injured neurons. Witmer-Pack et al. reported that microglial cells are not completely dependent on CSF-1, since they can be found in the brain. ${ }^{14}$ Quantitatively, it has been reported that during cerebral cortical ischemic lesion the numbers of microglia per $\mathrm{mm}^{2}$ on the contralateral side (which would also represent normal, uninjured numbers of microglia in that region) are similar between wild-type and op/op mice. However, around the injury side, the numbers of microglia remain unchanged in op/op mice, whereas those of wildtype animals increase by 2.9 fold. ${ }^{15}$

Accordingly, the op/op mouse offered us the opportunity to determine if the proliferation aspect of microglial activation plays a critical role in microglial-mediated excitotoxic neurodegeneration. 


\section{Results}

\section{Decreased proliferation but wild-type-like morphological activation of op/op microglia after kainic acid injection}

After injection of kainic acid into the hippocampus of control (C57/BI6) mice, microglial cells undergo activation. The kinetics of this activation process have been determined. ${ }^{1}$ Although the process of microglial activation begins shortly after injury, maximum levels of microglial activation, as evidenced by dramatic morphological changes, are reached

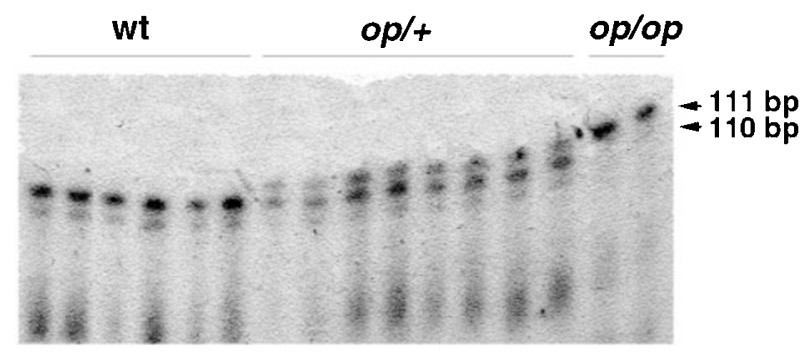

Figure 1 PCR-genotyping of op/op mice. Wild-type (C57/B/6), op/+, and op/ op mice were genotyped from tail DNA by a one-step PCR reaction as described in Materials and Methods. The PCR products were analyzed on a $6 \%$ polyacrylamide gel and visualized by autoradiography. C57/BI6 mice amplify a single 110 base pair product (see arrow), op/+ mice amplify 110 and 111 base pair products, and the op/op mice amplify a single 111 base pair product (see arrow)
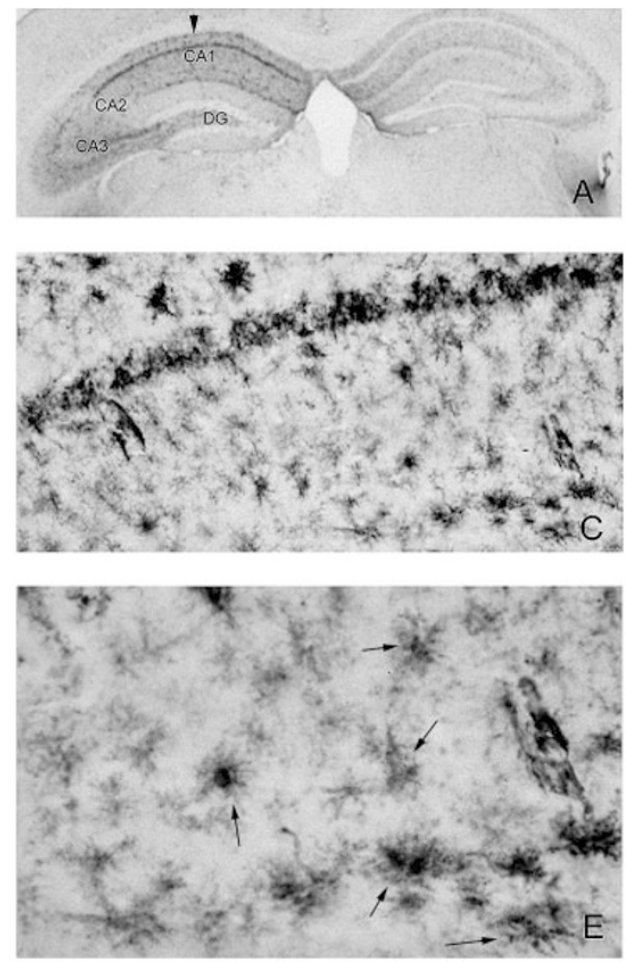

Figure 2 Microglial proliferation is deficient after excitotoxic injury, but microglial activation proceeds normally in op/op mice. Kainate was injected unilaterally into the hippocampi of control $(o p /+; n=11)$ and op/op mice $(n=12)$ to induce microglial responses (arrows indicate sites of injection on $\mathbf{A}$ and $\mathbf{B})$. Control mice respond with activation of their microglia on the injected side (C and $\mathbf{E}$ ). Such activation also includes a strong microglial proliferative response (seen best in $\mathbf{C}$ ). While the proliferation of microglia in the injected side of the hippocampus of the op/op mice is defective (seen best in D), a higher powered photomicrograph indicates that the microglia that migrate to the site of injury have the characteristics of activated microglia (D and F). The hippocampal subfields CA1, CA2 and CA3 as well as the dentate gyrus (DG) are labeled 
and F), the microglia in the control and op/op mice appeared similar aside from minor morphological differences visualized using F4/80 staining, as reported previously. ${ }^{16}$

To evaluate the levels of microglial proliferation after excitotoxin injection, we used double immunofluorescence against F4/80 (detecting microglia) and BrdU (for proliferating cells). As shown in Figure 3 , microglial proliferation was clearly evident in wild-type mice (wt), as well as in $\mathrm{tPA}^{-1-}$ ones (even though the overall number of microglial cells was significantly reduced). Proliferation was not observed for F4/ $80^{+}$microglia in op/op mice, as was earlier reported. ${ }^{15}$ Therefore, in this excitotoxin-injection model, microglia in op/ op mice display the phenotypic characteristics of microglial activation and are recruited to the sites of injury but do not mount a proliferative response (as evident by the presence of fewer activated $\mathrm{F} 4 / 80^{+}$cells and the observation that the $\mathrm{F} 4 / 80^{+}$ cells are not BrdU labeled). This observation is in agreement with the data obtained in the facial nerve axotomy model. ${ }^{13}$

\section{Pyramidal neurons in op/op mice undergo excitotoxic cell death}

We previously showed that the microglial activation normally observed in wild-type mice following kainate injection is

Table 1 Decreased numbers of activated microglia and lower levels tPA activity in the brains of op/op mice

\begin{tabular}{lcc}
\hline Genotype & $\begin{array}{c}\text { Activated microglia } \\
\text { in CA1 } \pm \text { s.d.* }\end{array}$ & $\begin{array}{c}\text { tPA (ng/ } / \mu \mathbf{g} \\
\text { protein } \pm \mathbf{s . d .})^{\star *}\end{array}$ \\
\hline Control & $73 \pm 7$ & $4.63 \pm 0.01$ \\
op/op & $26 \pm 10$ & $1.99 \pm 0.21$ \\
\hline
\end{tabular}

Activated microglia in the CA1 region of kainate-injected control (C57/B16) and op/op mice were counted as decribed in the text. Activated microglia were identified based on intensity of immunostaining by F4/80 and characteristic morphological changes. The data are presented as the average number of microglia present in four microscopic fields from cryostat coronal $14 \mu \mathrm{m}$ sections of brain around the injection site. Few if any activated microglia were present in the non-injected side in both control and op/op mice. Brains from control (C57) B16) and op/op mice were homogenized, as listed in the Materials and Methods section, and the levels of IPA activity were measured by the amidolytic assay at 30 and $90 \mathrm{~min}, 12$ and $24 \mathrm{~h}$. tPA activity was calculated from initial rates in the amidolytic assay. Extracts from $\mathrm{tPA}^{-1-}$ brains were used as negative controls. ${ }^{\star} P<0.01$, value associated with Student's two-tailed $t$-test. ${ }^{* *} P<0.05$, value associated with Student's two-tailed $t$-test significantly attenuated in tPA-deficient mice. ${ }^{9}$ Furthermore, the pyramidal neurons in $\mathrm{PPA}^{-/-}$mice, as well as in wild-type mice in which microglial activation has been delayed by macrophage-microglial inhibitory factor, ${ }^{2}$ are resistant to excitotoxin-induced neuronal death. To evaluate whether eliminating the proliferative component of the microglial response was sufficient to confer protection against neuronal cell death, we assessed neuronal survival in control (op/+) and op/op mice after the unilateral injection of kainate. As seen in Figure 4, the pyramidal hippocampal neurons were sensitive to excitotoxin injection in both genotypes, suggesting that there were sufficient activated microglia present in $o p /$ op mice to mediate neuronal death.

Combined with the results above, these data suggest that although microglia in op/op mice are unable to respond to CSF-1 by proliferating, they can respond to the signals produced by injured neurons to cause their activation and ultimately promote neuronal death.

\section{Decreased levels of tissue plasminogen activator in op/op mice}

Tissue plasminogen activator (tPA) is a serine protease that catalyzes the conversion of the zymogen plasminogen to the active protease plasmin. Both neurons and microglia in the mouse brain express this protease. The synthesis of tPA is rapidly upregulated after microglial activation in cell culture. We have previously shown that IPA can promote excitotoxic cell death in the mouse brain and suggested that secreted microglial-derived tPA may contribute to the neurotoxic properties of these cells. ${ }^{2}$ Given that the mutation that causes to the op/op phenotype results in defective proliferation of microglial cells only (but does not affect the numbers of neurons), we performed both zymographic and amidolytic assays to determine the levels of tPA. As shown in Table 1, there is a significant difference between the levels of tPA in the kainate-injected brains of control mice (4.63 ng of active tPA $/ \mu \mathrm{g}$ of brain extract), and op/op mice (1.99 ng of active $\mathrm{tPA} / \mu \mathrm{g}$ of brain extract) as demonstrated by the amidolytic assay. Similar differences in tPA levels were noted by zymographic analysis (data not shown). It is interesting to note in the op/op forebrain there is $\sim 43 \%$ tPA activity present compared to that of control mice, a number

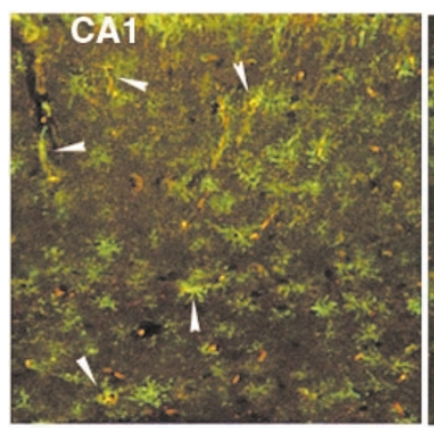

wt

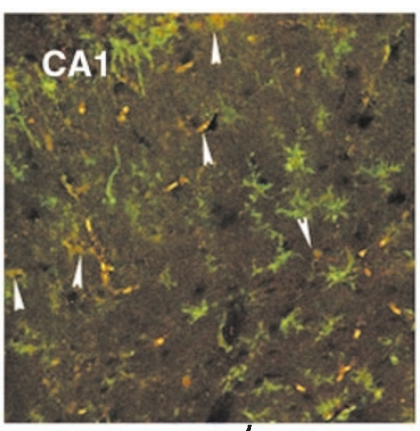

tPA $^{-/-}$

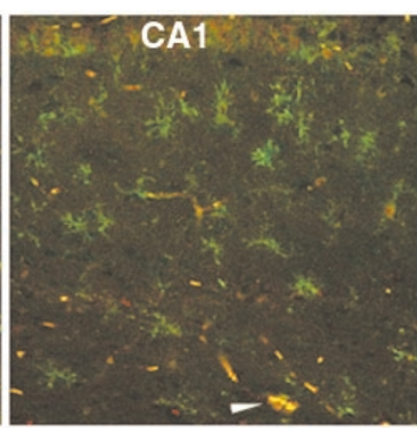

oplop

Figure 3 Microglial proliferation is nearly absent in op/op mice during excitotoxin-induced neuronal death. Microglial proliferation was evaluated by double immunofluorescence in wild-type, tPA-deficient and op/op mice on $14 \mu \mathrm{m}$ cryostat coronal sections. Arrows indicate the presence of doubly labeled (proliferating) microglia 

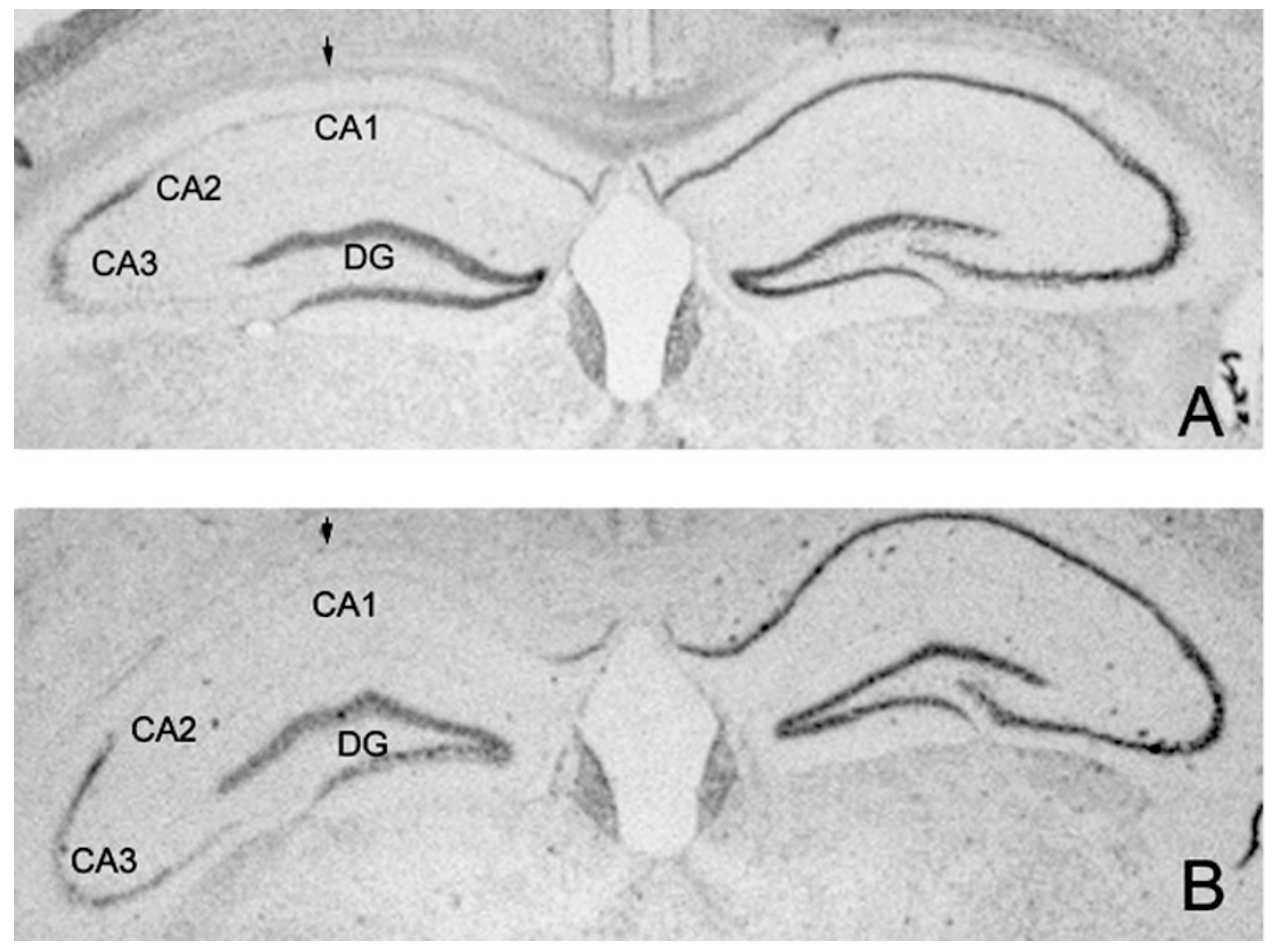

Figure 4 Op/op mice are sensitive to excitotoxin-induced neuronal death. The viability of pyramidal neurons in the hippocampus of injected control (op/+, $n=11$ ) and op/op mice $(n=12)$ after unilateral intrahippocampal kainate injection was evaluated by cresyl violet staining. In kainate-injected op/op mice $(B)$, the neurons at the injected side were eliminated to the same extent as those in control (B) mice. The arrows indicate the site of kainate injection. CA1, CA2 and CA3 indicate the hippocampal pyramidal subfields, and DG denotes the dentate gyrus of the hippocampal formation

close to the difference in activated microglia between the two genotypes (see Table 1). Since the amount of tPA correlated well with the numbers of microglia present in the injured hippocampus, and hippocampal neurons (the other producer of tPA in the mouse CNS parenchyma) were eliminated by the excitotoxin, we propose that IPA may be useful as a marker for microglial proliferation in the mouse forebrain.

\section{Discussion}

The op/op mouse carries a frameshift in the CSF-1 gene ${ }^{11}$ that results in defective microglial proliferation both in cell culture and in vivo. ${ }^{12,13}$ Previously, we reported that retarding microglial activation in response to excitotoxin injection could protect against neuronal death. ${ }^{2}$ This suggested a neurotoxic role for microglia in the mouse brain during excitotoxicity. However, it was uncertain which component(s) of microglial activation is necessary for excitotoxin-mediated cell death. We set out therefore to ascertain whether impairment of microglial proliferation would confer protection from neuronal death, or if the initial number of activated microglia present would suffice to promote neuronal death. Intrahippocampal delivery of kainic acid into the brain parenchyma of the op/op mice resulted in neuronal cell death, a result consistent with the observed vulnerability of $o p / o p$ cortical neurons to ischemic insult. ${ }^{15}$ These results suggest that despite the diminished proliferative capability of microglia in op/op mice, the microglia that become activated are able to promote neurotoxicity and to phagocytose the debris of injured neuronal cells. In addition it is suggested that a threshold may exist for the number of activated microglial cells above which they cease to become a rate-limiting factor in this neurodegeneration pathway.

These neurotoxic properties of microglia may be the result of the up-regulation and secretion of several factors that these cells express, such as tPA and TNF- $\alpha^{10,17}$ Interestingly, at least TNF- $\alpha$ (and potentially tPA) lies at the beginning of potent signal cascades that can result in cell death. ${ }^{18}$ This provides a rationale through which even a small number of activated microglia could provide the necessary downstream signals to effect neuronal cell death. We have already shown that the tPA/plasminogen proteolytic cascade promotes excitotoxic cell death. ${ }^{19,20}$ Furthermore, microglial-derived tPA may initiate this cascade (C-J Siao, personal communication). The data presented here demonstrate that the number of activated microglia is diminished in the kainate-injected op/op mouse (36\% of control), and these data correlate with the levels of decreased enzymatic activity of tPA in kainate-injected op/ op mice ( $43 \%$ compared to those of control mice). This result further suggests that microglial tPA is a major source of tPA locally at the site of injury. The potential for signal amplification present in a proteolytic cascade initiated by tPA and plasmin may account for the strength of the neurotoxic effects of the few activated microglia in the op/ op mouse. Moreover, we propose that tPA can be used as a marker for microglial proliferation following injury induced by kainate. 
Additionally, TNF- $\alpha$ has been implicated to play a role in the neurotoxic properties which microglia possess. ${ }^{21,22}$ TNF- $\alpha$ is secreted by activated microglia and can inhibit the re-uptake of glutamate by astrocytes, thereby allowing higher and potentially toxic concentrations of extracellular glutamate. ${ }^{21}$ TNF- $\alpha$ lies at the beginning of a biochemical cascade that potentiates neuronal death following kainate injection. ${ }^{18}$ In fact, we report here that there is approximately half the level of TNF- $\alpha$ present in the kainateinjected op/op mouse, yet excitotoxin-induced neurodegeneration proceeds normally. This may occur because TNF- $\alpha$ can stimulate the generation of IL-1 $\beta$, IL- 6 and other cytotoxic cytokines. ${ }^{23}$ However, we have also previously shown that in tPA-deficient mice (where microglial activation is attenuated $)^{9}$ neurons are resistant to excitotoxicity and the microglia in these mice secrete $43 \%$ less TNF- $\alpha$ than wild-type mice in response to activation stimuli. ${ }^{10}$ This amount of TNF- $\alpha$ is comparable to that secreted by op/op microglia. It is tempting to speculate that the different effects of kainate in tPA-deficient and op/op mice lie in the ability of activated microglia in op/op mice to produce sufficient tPA to cross a threshold and successfully initiate the biochemical cascades that lead to cell death.

Activated microglia have been implicated to play a role in several neuropathological conditions such as Alzheimer's disease, stroke and multiple sclerosis. Previously, Raivich et al. $^{13}$ reported that microglia in op/op mice underwent normal activation in the facial nerve axotomy model. In the present study we show that the activation of microglia, rather than their proliferation, is critical for effecting neuronal death. We and others previously demonstrated that microglia possess neurotoxic properties, ${ }^{2,5,6,24}$ and that inhibition of microglial activation can be neuroprotective against kainate-induced neuronal injury and death. Taken together with the data presented here, we suggest that the toxic mediators, secreted by microglia after neuronal injury, are sufficiently potent that even decreased numbers of activated microglia are able to promote neuronal death. Therefore, any potential neuroprotective therapy involving suppression of microglial activation must take their potency into account.

\section{Materials and Methods}

\section{Animal procedures}

All experiments performed on mice were done in accordance to the $\mathrm{NIH}$ guide for the care and use of laboratory animals as well as the institutional guidelines set by the IACUC Committee and the Division of Laboratory Animal Research at Stony Brook. All efforts were made to minimize the use of animals and to ensure minimal suffering of those animals used.

\section{Mice}

The osteopetrosis (op/op) mouse arose form a spontaneous mutation in the CSF-1 gene in C57/BI6 mice. This single base insertion within the coding region of CSF-1 leads to a truncated, non-functional protein. ${ }^{24}$ Since homozygote op/op mice do not breed well, op/+ heterozygotes were mated to expand the op/op colony. The genotypes of the offspring were determined using a PCR-based assay. Primers that flanked the mutation were designed (primer 1: $5^{\prime}$-CAGCTGGATGATCCTGTTTGC-3'; primer 2: 5'-CTCGGTGGCGTTAGCATTGGG$3^{\prime}$ ) such that genomic DNA from a homozygote op/op mutant would yield a 111 base pair product whereas wild-type DNA would yield a 110 base pair product. Genomic DNA was prepared from the tails of mice using standard procedures. The genomic DNA was PCR-amplified using the above mentioned primers using the following protocol: $94^{\circ} \mathrm{C}$ $5 \mathrm{~min}$ followed by 30 cycles of $94^{\circ} \mathrm{C} 45 \mathrm{sec}, 58^{\circ} \mathrm{C} 1 \mathrm{~min}, 72^{\circ} \mathrm{C} 1 \mathrm{~min}$, with a final extension at $72{ }^{\circ} \mathrm{C}$ for $7 \mathrm{~min} .{ }^{32} \mathrm{P}$-dATP was included in the PCR reactions. The products were separated on a $6 \%$ polyacrylamide gel and subjected to autoradiography to visualize the bands (Figure 1).

\section{Intrahippocampal injection of kainate}

Adult C57BI6/J, op/+ and op/op male mice, between 20 and 25 grams, were injected intraperitoneally with atropine $(0.6 \mathrm{mg} / \mathrm{kg}$ of body weight) and deeply anesthetized with $2.5 \%$ avertin $(0.02 \mathrm{ml} / \mathrm{gram}$ of body weight). The mice were then injected with $1.5 \mathrm{nmol}$ kainic acid (in $300 \mathrm{nl}$ phosphate buffered saline) unilaterally into the hippocampus using stereotaxic coordinates (bregma $-2.5 \mathrm{~mm}$, medial/lateral $1.7 \mathrm{~mm}$ and dorsoventral $1.6 \mathrm{~mm}$ ). The excitotoxin was delivered over $30 \mathrm{sec}$, and the injection needle remained in place for two additional min to prevent reflux of fluid. Five days after the injection, the brains of the injected mice were analyzed for neuronal survival and microglial activation.

\section{BrdU labeling of proliferating microglial cells}

At $48 \mathrm{~h}$ following kainate injection, BrdU $(200 \mathrm{mg} / \mathrm{kg})$ was delivered to the mice by intraperitoneal injection. These injections occurred once daily for 3 days. At day 5 post-kainate injection, the mice were sacrificed and subjected to immunohistochemistry as described below.

\section{Immunohistochemistry}

Coronal sections $(30 \mu \mathrm{m})$ of the brains of the injected mice were cut at the level of the hippocampus. Non-specific immunoreactivity was blocked by incubation of the brain sections with $5 \%$ goat serum. The sections were incubated with antibodies either to the mature macrophage/microglia specific antigen F4/80 (1:100, Serotec) or Mac-1 (1:10, Roche Biochemicals). Biotinylated secondary antibodies were used (Vector Laboratories) and the avidin-biotin-peroxidase complex ( $A B C$ reaction) was visualized with diaminobenzidine and hydrogen peroxide (Vector Laboratories) as described previously. ${ }^{20}$ For BrdU immunostaining the tissue sections were treated with $2 \mathrm{~N} \mathrm{HCl}$ prior to preincubation with goat serum. Fluorescent-labeled secondary antibodies (anti-rat FITC for F4/80 and anti-mouse TRITC for BrdU) were used to identify proliferating microglial cells.

\section{Amidolytic assay for tPA activity}

For quantitative determination of tPA activity, the amidolytic assay was performed as previously described. ${ }^{25}$ Briefly, the tissue was lysed in $0.25 \%$ Triton $\mathrm{X}-100$ and incubated at $25^{\circ} \mathrm{C}$ in a mix containing $0.3 \mathrm{mM}$ S-2251 and $0.42 \mu \mathrm{M}$ plasminogen in $0.1 \mathrm{M}$ Tris, $\mathrm{pH} 8.1,0.1 \%$ Tween$80)$. The change in absorbance $(\Delta \mathrm{A})$ at $405 \mathrm{~nm}$ was measured at different time-points. Known concentrations of recombinant IPA protein were used to generate a standard curve.

\section{Quantitation of microglial cell numbers}

$\mathrm{F} 4 / 80^{+}$and $\mathrm{Mac}-1^{+}$microglia in the CA1 hippocampal subfield were counted in four successive cryostat coronal sections (cut at $14 \mu \mathrm{m}$ ) 
around the injection site, and the numbers were averaged, as previously described. ${ }^{26}$ Microglial cell counting occurred 5 days after the excitotoxic injury, when microglial activation (evident by F4/80 immunostaining) reaches peak levels. ${ }^{1}$ Activated microglia were characterized as cells with a cell body larger than $10 \mu \mathrm{m}$ in diameter, with short, thick processes and intense immunoreactivity. No resting microglia (characterized by small cell body, long processes and weak immunoreactivity) were observed at that time-point on the sections.

\section{Quantification of microglial activation}

Whole brain lysates were prepared by dounce homogenization in phosphate buffered saline from kainate-injected control and op/op mice. To estimate the relative amounts of activated microglia present in each brain, relative tumor necrosis factor alpha (TNF- $\alpha$ ) levels were assessed by Western blot analysis. Briefly $20 \mu \mathrm{g}$ of total lysate was separated through a $15 \%$ polyacrylamide gel and transferred to a PDVF membrane. TNF- $\alpha$ was detected using a rat-anti-mouse TNF- $\alpha$ antibody (clone MP6-XT3) at a dilution of 1:500 (Boehringer Mannheim) followed by biotinylated goat-anti-rat secondary antibody at a 1:3000 dilution (Vector Laboratories). Finally, the avidin-biotinperoxidase complex (ABC Elite, Vector Laboratories) was visualized by chemiluminescence (LumiGLO, KPL). The relative amounts of TNF$\alpha$ were determined using a Bio-Rad densitometer.

\section{Acknowledgements}

We would like to thank Dr. S Strickland for advice, and are grateful to Dr. $M$ Frohman for critical reading of the manuscript and the members of the Tsirka laboratory for helpful advice and discussions. This work was supported by fellowships from the Medical Scientist Training Program (AD Rogove), National Institutes of Health and an Army Medical Research Grant (SE Tsirka).

\section{References}

1. Andersson P, Perry V and Gordon S (1991) The kinetics and morphological characteristics of the macrophage-microglial response to kainic acid-induced neuronal degeneration. Neuroscience 42: 201-214

2. Rogove A and Tsirka S (1998) Neurotoxic responses by microglia elicited by excitotoxic injury in the mouse hippocampus. Curr. Biol. 8: 19-25

3. Lipton S and Rosenberg P (1994) Mechanisms of disease: Excitatory amino acids as a final common pathway for neurological disorders. New Eng. J. Med. 613-622

4. Wang Y, Tsirka S, Strickland S, Stieg P, Soriano S and Lipton S (1998) Tissue plasminogen activator (TPA) increases neuronal damage after focal cerebral ischemia in wild-type and tPA-deficient mice. Nature Med. 4: 228-232

5. Wood P (1995) Microglia as a unique cellular target in the treatment of stroke: potential neurotoxic mediators produced by activated microglia. Neurological Research 17: 242-248

6. Giulian D, Vaca K and Corpuz M (1993) Brain glia release factors with opposing actions upon neuronal survival. J. Neurosci. 13: 29-37

7. Heyes M, Achim C, Wiley C, Major E, Saito K and Markey S (1996) Human microglia convert L-tryptophan into the neurotoxin quinolinic acid. Biochem. $\mathrm{J}$. 320: 595-597
8. Walker D, Kim S and McGeer P (1995) Complement and cytokine gene expression in cultured microglia derived from postmortem human brains. J. Neurosci. Res. 40: 478-493

9. Tsirka S, Gualandris A, Amaral D and Strickland S (1995) Excitotoxin induced neuronal degeneration and seizure are mediated by tissue-type plasminogen activator. Nature 377: $340-344$

10. Rogove A, Siao C-J, Keyt B, Strickland S and Tsirka S (1999) Activation of microglia reveals a non-proteolytic cytokine function for tissue plasminogen activator in the central nervous system. J. Cell Sci. 112: 4007-4016

11. Yoshida H, Hayashi S-I, Kunisada T, Ogawa M, Nishikawa S, Okamura H, Sudo T, Schultz L and Nishikawa S-I (1990) The murine mutation osteopetrosis is in the coding region of the macrophage colony stimulating factor gene. Nature 345 : $442-444$

12. Blevins $G$ and Fedoroff $S$ (1995) Microglia in colony stimulating factor 1-deficient op/op mice. J. Neurosci. Res. 40: 535-544

13. Raivich G, Moreno-Flores T, Moller J and Kreutzberg G (1994) Inhibition of posttraumatic microglial proliferation in a genetic model of macrophage colonystimulating factor deficiency in the mouse. Eur. J. Neurosci. 6: 1615-1618

14. Witmer-Pack M, Hughes D, Schuler G, Lawson L, McWilliam A, Inaba K, Steinman R and Gordon S (1993) Identification of macrophages and dendritic cells in the osteopetrosis (op/op) mouse. J. Cell Sci. 104: 1021-1029

15. Berezovskaya O, Maysinger D and Federoff $S$ (1995) The hematopoietic cytokine, colony-stimulating factor 1 , is also a growth factor in the CNS: congenital absence of CSF-1 in mice results in abnormal microglial response and increased neuron vulnerability to injury. Int. J. Dev. Neurosci. 13: 285-299

16. Wegiel J, Wisniewski HM, Dziewiatkowski J, Tarnawski M, Kozielski R, Trenkner E and Wiktor-JedrzejczakW (1998) Reduced number and altered morphology of microglial cells in colony stimulating factor-1-deficient osteopetrotic op/op mice. Brain Res. 804: 135-139

17. Kreutzberg G (1996) Microglia: a sensor for pathological events in the CNS. Trends Neurosci. 19: 312-318

18. Chao CC and Hu S (1994) Tumor necrosis factor-alpha potentiates glutamate neurotoxicity in human fetal brain cell cultures. Developmental Neuroscience 16: $172-179$

19. Tsirka S, Rogove A and Strickland S (1996) tPA and neuronal death. Nature 384: $123-124$

20. Tsirka S, Rogove A, Bugge T, Degen J and Strickland S (1997) An extracellular proteolytic cascade promotes neuronal degeneration in the mouse hippocampus. J. Neurosci. 17: 543-552

21. Fine S, Angel R, Perry S, Epstein L, Rothstein J, Dewhurst S and Gelbard H (1996) Tumor necrosis factor alpha inhibits glutamate uptake by primary human astrocytes - implications for pathogenesis of HIV-1 dementia. J. Biol. Chem. 271: $15303-15306$

22. Yoshimoto T, Houkin K, Tada M and Abe H (1997) Induction of cytokines, chemokines and adhesion molecule mRNA in a rat forebrain reperfusion model. Acta Neuropathol. 93: 154-158

23. Lee S, Liu W, Dickson D, Brosnan C and Berman J (1993) Cytokine production by human fetal microglia and astrocytes. Differential induction by lipopolysaccharide and IL-1 beta. J. Immunol. 150: 2659-2667

24. Giulian D and Robertson C (1990) Inhibition of mononuclear phagocytes reduces ischemic injury in the spinal cord. Ann. Neurol. 27: 33-42

25. Andrade-Gordon P and Strickland S (1986) Interaction of heparin with plasminogen activators and plasminogen: effects on the activation of plasminogen. Biochemistry 25: 4033-4040

26. Batchelor P, Liberatore G, Wong J, Porritt M, Frerichs F, Donnan G and Howells D (1999) Activated macrophages and microglia induce dopaminergic sprouting in the injured striatum and express brain-derived neurotrophic factor and glial cell line-derived neurotrophic factor. J. Neurosci. 19: 1708-1716 